\title{
Efecto de la actividad gráfica en las emociones y el estrés
}

\author{
Minerva VANEGAS-FARFANO ${ }^{1}$ \\ Universidad Autónoma de Nuevo León, Nuevo León, México \\ minerva.vanegas@gmail.com \\ Mónica GONZÁLEZ ${ }^{2}$ \\ Universidad Autónoma de Nuevo León, Nuevo León, México \\ monygzz77@yahoo.com
}

Recibido: 09/01/14

Aceptado: 02/11/14

\section{RESUMEN}

Estudio que compara el uso de las actividades artísticas en alumnos universitarios $(\mathrm{n}=20)$ para regular las emociones y el estrés, bajo las condiciones de distracción y enfoque hacia un evento estresante. La condición experimental fue inducida recordando un evento estresante actual de los participantes, asignados a una de cuatro intervenciones. Los niveles de estrés y afecto fueron medidos antes y después de realizar la actividad asignada. Los resultados revelan disminución significativa del estrés y el afecto negativo bajo la condición de distracción. El resultado indica una posible asociación entre el uso de la actividad artística como herramienta ante el estrés y las estrategias de afrontamiento de uso regular.

Palabras clave: Actividad artística, Afrontamiento del estrés, Regulación emocional.

\section{Referencia normalizada}

VANEGAS M., GONZÁLEZ M. (2014). "Efecto de la actividad gráfica en las emociones y el estrés". En Arteterapia: Papeles de arteterapia y educación artística para la inclusión social Vol.: 9. Páginas 145-154. Madrid.

\section{SUMARIO}

Introducción teórica. Aproximación metodológica. Resultados. Discusión. Referencias bibliográficas.

\section{Art-making effect on emotions and stress}

\footnotetext{
${ }^{1}$ Estudiante del Doctorado en Psicología por la UANL, egresada de la Maestría en Arte Moderno y Contemporáneo por Casa Lamm. Universidad Autónoma de Nuevo León, UANL, Facultad de Psicología. Av. Universidad S/N Ciudad Universitaria San Nicolás de los Garza Nuevo León, C.P. 66451 México.

${ }^{2}$ Doctora en Psicología por la UNED, España. Máster en Ciencias y Licenciada en Psicología por la UANL. Miembro del Sistema Nacional de Investigadores (S.N.I. - Nivel I). Universidad Autónoma de Nuevo León, UANL, Facultad de Psicología. Av. Universidad S/N Ciudad Universitaria San Nicolás de los Garza Nuevo León, C.P. 66451 México.
} 


\begin{abstract}
Two ways of art-making (distraction and expression of the event) are examined as a form of stress and mood regulation activity in undergraduate students $(n=20)$. A present stressful situation is recalled by the participants as an induced situation and the assigned to one of four conditions. Stress and affect were mesured before and after de assigned activity. Results reveals that stress and negative affect improved significantly in the distraction condition. The result indicates a probably association between art-making as a stress management activity and regular coping strategies.
\end{abstract}

Key words: Art-making, Stress coping, Emotion regulation.

\title{
CONTENTS
}

Theoretical introduction. Methodological approach. Results. Discussion. References.

\section{Introducción teórica}

Con objetivos como la reducción de síntomas o el incremento del bienestar personal y la efectividad, las terapias psicológicas datan desde hace muchos años. A través del tiempo se han desarrollado diversos modelos teóricos y técnicas de intervención en relación a los síntomas u objetivos terapéuticos considerando el contexto del individuo como las posibilidades que éste y el terapeuta tienen como herramientas para apoyarle.

La actividad artística es reconocida por muchos como una forma de expresión de emociones y pensamientos. Ésta, frente a la necesidad de mejoras en el apoyo a la salud mental ha dado lugar a un grupo de intervenciones denominadas terapias artísticas o expresivas cuyo espacio de trabajo incluye tanto el manejo en ámbitos hospitalarios como no clínicos.

Con apenas una marcada proliferación como práctica clínica a partir de la era moderna y hasta nuestros días (Camic, 2008), la actividad artística presenta a la fecha resultados sobre sus efectos en la salud psicológica con un amplio rango de beneficios como el desarrollo de sentimientos de libertad, apoyo en la consecución de retos y logros, el proveer de nuevas relaciones sociales y generar cambios en el significado de las vivencias; además de brindar apoyo en las evaluaciones diagnósticas y procesos de recuperación de diversas enfermedades (Camic, 2008).

A pesar de la proliferación de estrategias para las intervenciones la profesionalización de la práctica en arte terapia se ha visto rezagada por numerosos problemas en varios países, incluyendo a México. Uno de estos es el desconocimiento de la manera en que ésta permite dichos cambios. Tal incapacidad para aportar suficiente sustento a los beneficios reportados incluye la elección del método de estudio, por una aparente preferencia del uso de estudios cualitativos frente a los cuantitativos (Read, 2009); a pesar del paulatino incremento en el área de diseños cuasi-experimentales, los cuales se centran generalmente en la confirmación del dato que avala el beneficio, mas no en la búsqueda o reconocimiento de aquellas variables que intervienen en la dinámica arte-salud (Slayton, 
D'Archer y Kaplan, 2010). Otro aspecto que ha sido enfatizado es la falta de un enfoque teórico desde el cual sea posible interpretar el dato (Read, 2009).

Toda intervención terapéutica requiere del dato que avale su eficacia. Para ello, el reconocimiento de las causas o elementos indispensables que permitan explicar cómo incide la actividad terapéutica en la salud psicológica se presenta como un punto a acometer para el cambio. En el caso de la terapia artística, siendo el arte un producto que integra diversos elementos como son los visuales, auditivos, audiovisuales, táctiles, entre otros, la no delimitación de sus diversos componentes eliminan la capacidad discriminativa del estudio (Wood, Molassiotis \& Payne, 2011; Read, 2009).Así mismo, la marcada diferencia entre la cantidad de estudios realizados en poblaciones con diagnósticos como la enfermedad mental, la neurológica o la crónica, o bien en etapas de edad temprana o en la vejez, frente a aquellos con población abierta es otra situación que limita el reconocimiento de sus beneficios en otros grupos de la población (Wood, Molassiotis \& Payne, 2011).

\section{Las terapias psicológicas y su vínculo con el manejo del estrés y las emocio- nes}

El estrés es un término conocido en la actualidad aun por aquellos cuyas actividades no se vinculan en el mantenimiento o la mejora de la salud mental. Como fenómeno común para la vida cotidiana, sus efectos en ésta son ampliamente conocidos y estudiados, al igual que sus vínculos con otras variables que inciden en la salud psicológica de las personas. Por ejemplo, en el caso de las emociones cuando éstas son afrontadas efectivamente se logra una mediación entre el estrés y el desarrollo de síntomas psicopatológicos (Berking, Poppe, Luhmann, Wupperman, Jaggi y Seifritz, 2012; Szczygiel, Buczny, y Bazinka, 2012).

Teniendo como base la teoría transaccional del estrés de Lazarus y Folkman(1987), su relación con las emociones y sobre todo, los aportes del vínculo entre ambas como elemento clave para mantener la salud psicológica, el presente incluye los resultados de un estudio piloto cuyo proyecto de origen mantiene como objetivo general evaluar el uso de las actividades artístico/recreativas como una forma de manejo de las emociones y afrontamiento del estrés. Los objetivos específicos el presente estudio buscan:

1) Evaluar el efecto en el nivel de estrés percibido al utilizar actividades artísticos/recreativas como forma de afrontamiento del estrés.

2) Evaluar si al utilizar actividades artístico/recreativas, con exclusiva representación gráfica o con una explicación narrada de lo realizado, funcionan como un distractor de las situaciones estresantes.

3) Evaluar si al utilizar actividades artístico/recreativas, con exclusiva representación gráfica o con una explicación narrada de lo realizado, funcionan como una forma de afrontamiento activa (solución de problemas) para las situaciones estresantes.

4) Evaluar el efecto en las emociones al utilizar actividades artísti$\cos /$ recreativas como forma de afrontamiento del estrés. 
5) Comparar cambios en las emociones (positivas y negativas) al utilizar actividades artístico/recreativas, con exclusiva representación gráfica o con una explicación narrada de lo realizado, cuando son utilizadas como distractores de las situaciones estresantes.

6) Comparar cambios en las emociones (positivas y negativas) al utilizar actividades artístico/recreativas, con exclusiva representación gráfica o con una explicación narrada de lo realizado, como una forma de afrontamiento activa (solución de problemas) para las situaciones estresantes.

\section{Aproximación metodológica}

Muestra. La población de donde se derivó la muestra fue la de estudiantes universitarios, ya sobre éstas no se han reportado artículos que aborden intervenciones artísticas para la reducción del estrés. Se seleccionó aleatoriamente un grupo del turno matutino de una facultad de odontología, en donde se pidió la participación voluntaria y anónima de veinte estudiantes. Fueron considerados como criterios de inclusión: a) el estar inscrito en al menos la mitad de las materias consideradas como carga completa, b) estar capacitado para realizar actividades gráficas (dibujo) a nivel no profesional y c) poder identificar una situación estresante actual que influya actualmente en su vida. Como criterio de exclusión se utilizó el encontrarse en una o varias de las siguientes situaciones: a) periodo de duelo, ya sea por un familiar o amistad cercano; tener confirmado un diagnóstico de ansiedad, depresión o estrés post-traumático al momento del estudio y/o presentar un problema de abuso de sustancias.

Diseño. El proyecto se realizó mediante un diseño experimental factorial mixto orientado al aislamiento de los efectos de la actividad artística. Dado que se buscaba comparar los cambios en las variables dependientes; y teniendo en cuenta el efecto de regresión, su diseño incluyó medidas pre test y post test. A fin de controlar los efectos propios de la demanda de la actividad y teniendo en cuenta los comentarios recabados en la documentación sobre los usos de las actividades artísticas en ambientes terapéuticos, fueron consideradas dos condiciones de intervención: el trabajo gráfico/artístico como una distracción y la elaboración gráfico/artística como una herramienta de enfoque en la situación objetivo (estresante). Con la finalidad de estudiar la interacción entre el empleo de la actividad gráfica bajo ambas condiciones, y su relación con el uso de la narrativa del suceso (actividad común tanto en ésta como otro tipo de intervención terapéutica), se implementó un diseño en donde los sujetos, asignados al azar en grupos equivalentes, trabajaran en una de las cuatro combinaciones de tratamiento:

GRUPO $\mathrm{a}_{1} \quad$ Actividad como distractor + Narrativa de suceso

GRUPO $\mathrm{a}_{2} \quad$ Actividad como distractor + Sin narrativa del suceso

GRUPO $a_{3} \quad$ Actividad como enfoque + Narrativa de suceso

GRUPO $\mathrm{a}_{4} \quad$ Actividad como enfoque + Sin narrativa del suceso 
Instrumentos. Tras la revisión bibliográfica y en función a las variables de estudio, los objetivos y el marco teórico considerado, fueron seleccionados los siguientes instrumentos:

1. La Adaptación mexicana de la Escala de Estrés Percibido (PSS; Cohen, Kamarak y Mermelstein, 1983) realizada por González y Landero (2007). Ésta consta de catorce ítems con puntuación de nunca (0) a muy a menudo (4), con coeficiente de fiabilidad de .83

En el presente trabajo los resultados de fiabilidad del alfa de Cronbach demostraron una aceptable consistencia interna en el pretest $(\alpha=.84)$ y post test $(\alpha=.74)$

2. La adaptación española del Inventario de Estrategias de Afrontamiento, realizado por Cano, Rodríguez y García (2007). Ésta recoge tanto información cualitativa como cuantitativa, permitiendo realizar un análisis narrativo que profundice en el significado individual de la situación estresante. En su componente cuantitativo presenta una estructura jerárquica con ocho estrategias primarias, cuatro secundarias y dos terciarias. Dicha adaptación cuenta con cuarenta ítems y emplea una escala tipo Likert de cinco puntos. Su versión original presentó coeficientes de confiabilidad dentro del rango de .72 a .94 . En el presente trabajo el resultado de fiabilidad del alfa de Cronbach de la escala general mostró una aceptable consistencia interna, $\alpha=.85$

3. La Escala de Afecto Positivo y Negativo (PANAS; Watson y Clark, 1988), en su validación y versión corta en castellano para población mexicana realizada por Robles y Páez (2003). La cual mide las dos dimensiones de emocionalidad, afecto positivo y negativo, está compuesta por veinte reactivos divididos en dos apartados, diez para medir el afecto positivo y diez para el afecto negativo. La versión utilizada fue aquella que valora los sentimientos y emociones en la última semana, con una fiabilidad interna de .85 a.81. En el presente estudio los resultados de fiabilidad del alfa de Cronbach demostraron una aceptable consistencia interna en el pretest $(\alpha=.86)$ y post test $(\alpha=.86)$.

4. Imágenes del Sistema Internacional de Fotografías Afectivas. Cuya normatividad y desarrollo es exclusivo para lograr estímulos experimentales de emociones y atención. Fueron utilizadas imágenes de entornos e interiores urbanos cuyas medias y desviaciones estándar relacionadas al placer, estimulación y dominancia las ubicasen en la categoría de neutras de acuerdo al material proporcionado por el mismo Center for the Study of Emotion and Attention (s.f.).

Procedimiento. Al inicio de la intervención fue dada a conocer la naturaleza voluntaria y anónima del estudio y sus criterios de inclusión/exclusión; información que también se entregó impresa en un formato de consentimiento que contenía una ficha con datos sobre el procedimiento y riesgos o incomodidades derivados de la participación en el mismo. El material de trabajo, dos grupos de cuestionarios ordenados de acuerdo al tipo de intervención por grupo, una hoja tamaño carta de papel fabriano, doce gises pastel de colores y un lápiz con borrador, se incluyeron en un sobre cerrado, el cual presentaba la carta de consentimiento informado anteriormente descrita en su parte frontal. Los grupos de cuestionarios tenían impreso en la parte superior derecha un número correspon- 
diente al orden de ejecución, así mismo, fueron distinguidos con un color diferente los sobres de acuerdo al tipo de intervención. Estos se entregaron a los participantes de manera aleatoria, a quienes se les indicó que los colores de los sobres correspondían a un orden distinto de los inventarios y los tipos de actividad (dibujo) que se solicitaría. Al terminar de contestar los datos del primer grupo de cuestionarios se daba la indicación de realizar la actividad gráfica con el material que contenía el sobre, enfatizando en dos aspectos, de acuerdo al color que distinguía al paquete: "copiar una de las imágenes incluida en el material" la cual fue solicitada a The Center for the Study of Emotion and Attention (s.f.) e impresa para uso exclusivo del estudio o bien " representar el objeto o situación estresante que haya documento en los cuestionarios anteriores, sin importar que tan perfecto o bello quede el dibujo". En un tercer momento fue solicitado que se llenase el segundo grupo de inventarios (post test) y por último, considerando la necesidad de eliminar residuos de emociones negativas en torno a los sucesos estresantes señalados, se pidió a los participantes que realizaran una lista o bien un dibujo de "actividades que puedan realizar a fin de obtener resultados positivos a la situación estresante recordada, de acuerdo a sus habilidades actuales" o bien de "posibles desenlaces positivos a la situación estresante, de acuerdo a sus habilidades actuales". Posteriormente se solicitó que entregasen el material dentro del mismo paquete, incluyendo las imágenes proporcionadas para la intervención como distracción.

Análisis de datos. Se calcularon los estadísticos descriptivos, media y desviación típica, en

\section{Resultados}

Los datos sociodemográficos de los participantes se muestran en la tabla 1.A1 comparar los resultados de los inventarios utilizados antes de la intervención pudo observarse que no existían diferencias significativas entre los grupos, lo que corroboró la utilidad del método de muestreo aleatorio (Tabla 2).

\begin{tabular}{|c|c|c|c|c|}
\hline & Grupo 1 & Grupo 2 & Grupo 3 & Grupo 4 \\
\hline Edad & 21.4 (D.E. $=1.14$ ) & 20.8 (D.E. $=1.3)$ & 21 (D.E. $=.001)$ & 21.20 (D.E. $=1.09$ ) \\
\hline Sexo & $\begin{array}{l}\mathrm{H}=2 \\
\mathrm{M}=3\end{array}$ & $\begin{array}{l}\mathrm{H}=1 \\
\mathrm{M}=4\end{array}$ & $\begin{array}{l}\mathrm{H}=1 \\
\mathrm{M}=4\end{array}$ & $\begin{array}{l}\mathrm{H}=1 \\
\mathrm{M}=4\end{array}$ \\
\hline $\begin{array}{l}\text { Semestre } \\
\text { Ocupación }\end{array}$ & $\begin{array}{c}7 \mathrm{mo} . \\
\text { Estudiante }(\mathrm{N}=5)\end{array}$ & $\begin{array}{c}\text { 7mo. } \\
\text { Estudiante }(\mathrm{N}=3) \\
\text { Estudia y Trabaja }(\mathrm{N}=2)\end{array}$ & $\begin{array}{c}\text { 7mo. } \\
\text { Estudiante }(\mathrm{N}=2) \\
\text { Estudia y Trabaja }(\mathrm{N}=3)\end{array}$ & $\begin{array}{c}7 \mathrm{mo} . \\
\text { Estudiante }(\mathrm{N}=4) \\
\text { Estudia y Trabaja }(\mathrm{N}=1)\end{array}$ \\
\hline
\end{tabular}

Tabla 1

Tabla 2.

Resultados de las puntuaciones de los inventarios de estrés y emociones.

\begin{tabular}{|c|c|c|c|c|c|}
\hline $\begin{array}{l}\text { Estrés } \\
\text { Emociones }\end{array}$ & $\begin{array}{c}\text { Grupo 1 } \\
21.6(\text { D.E. }=7.3)\end{array}$ & $\begin{array}{c}\text { Grupo 2 } \\
26.8 \text { (D.E. }=4.49)\end{array}$ & $\begin{array}{c}\text { Grupo 3 } \\
27.6(\text { D.E. }=11.41)\end{array}$ & $\begin{array}{c}\text { Grupo } 4 \\
28.8(\text { D.E. }=4.81)\end{array}$ & $\begin{array}{c}\text { Kruskal-Wallis } \\
\mathrm{H}(3)=3.75, p=0.289\end{array}$ \\
\hline Positivas & $29.8($ D.E. $=7.7)$ & 32 (D.E. $=12.6)$ & $27.4($ D.E $=10.45)$ & 33.2 (D.E. = 7.6) 25.2 & $\mathrm{H}(3)=0.91, p=0.82$ \\
\hline
\end{tabular}

Tabla 2 
Tabla 3. Resuledos de las intervenciones por gnupo de acuerdoa los objetivos del estudio.

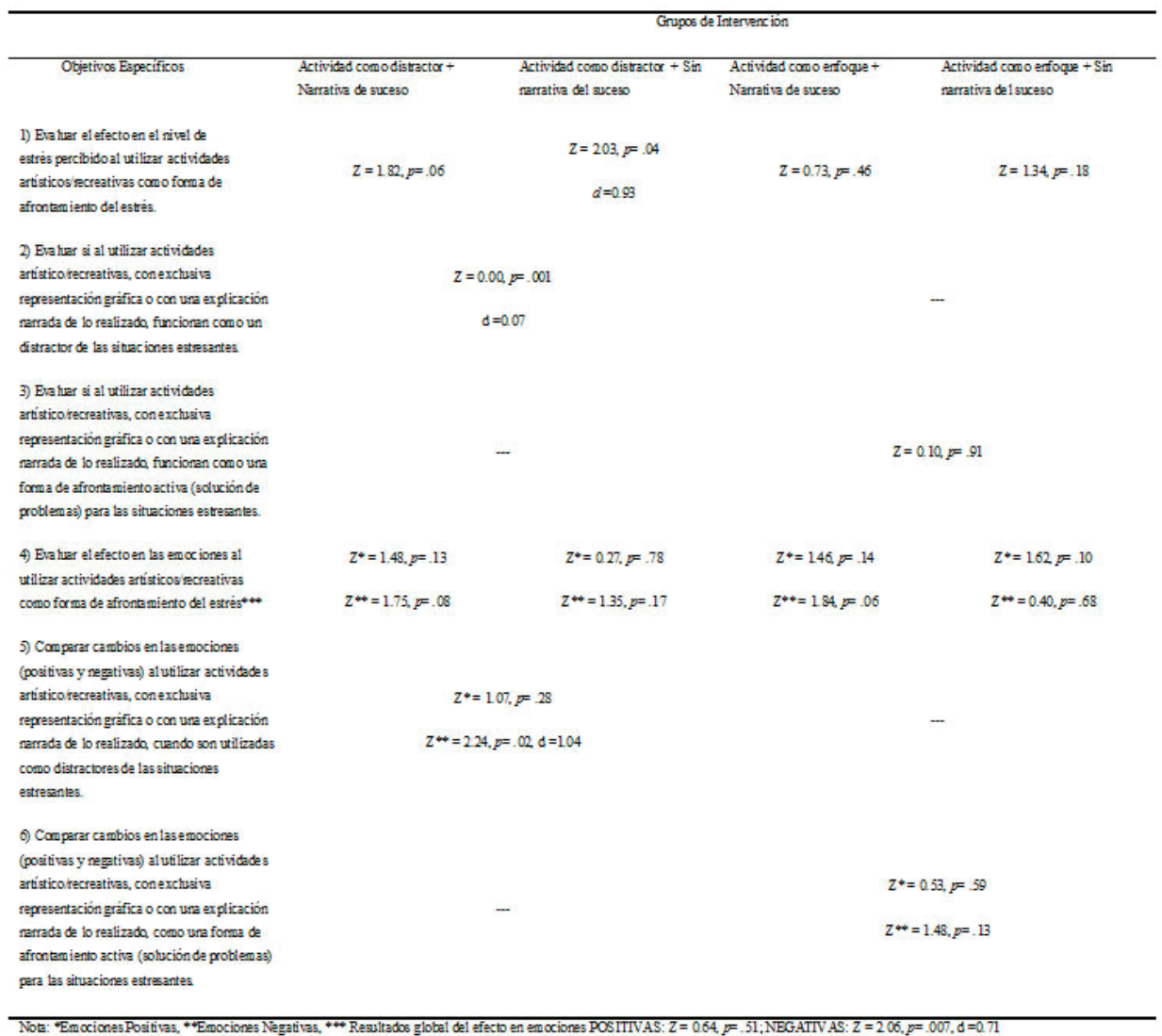

Tabla 3

Los resultados obtenidos en cada uno de los grupos, tras la intervención fueron:

Estrés. Al valorar cambios en el nivel de estrés percibido de las personas, ya sea enfocándose en la situación problemática o tratando de distraerse de ésta. Se obtuvo significancia estadística en la segunda condición de estudio, el distraerse de la situación $(\mathrm{Z}=0.20, p=.83)$.La variación estadísticamente significativa en el nivel de estrés percibido tras realizar la actividad señalada se concentró en el grupo al cual se le pidió que piense en una situación estresante actual, se distraiga realizando un dibujo con valencia emocional neutra; y posteriormente describa la situación sobre la cual se había concentrado (tabla 3). El uso de la actividad gráfica como forma de afrontamiento activa (enfoque en la situación estresante), no presentó significancia estadística en sus resultados.

Emociones. Al valorar cambios en las emociones de los participantes, se obtuvo significancia estadística en el caso de las emociones negativas al comparar 
los resultados de toda la muestra $(\mathrm{n}=20)$, es decir, en los grupos en general: $\mathrm{Z}=$ $2.06, p=.007, \mathrm{~d}=0.71$ ", no así en las emociones positivas las cuales presentaron como resultado $\mathrm{Z}=0.64, p=.51$. La significancia estadística se concentró en el grupo de aquellas personas que participaron en la condición de "uso de actividad gráfica como distractor". No se encontraron cambios estadísticamente significativos en las emociones de las participantes al trabajar la actividad artística como enfoque (tabla 3).

Estrategias de afrontamiento. Tras considerar que la prueba efectuada permitía indagar sobre el tipo de estrategia que utiliza la persona frente a la situación estresante, ya sea centrada en la emoción o la actividad, se procedió a efectuar el análisis de las distintas estrategias, considerando la estructura jerárquica de tres niveles proporcionada por Cano, Rodríguez y García (2007); en éste únicamente se obtuvo resultado estadísticamente significativo en el uso del pensamiento desiderativo como estrategia frente al estrés, a un nivel general: $\mathrm{M}=12.05$, D.E. $=5.3, p=.017,(\mathrm{n}=20)$

\section{Discusión}

La actividad artística, utilizada como herramienta para el apoyo de la salud psicológica se extiende poco a poco tanto en conocimiento como práctica. Los datos del presente proyecto indican congruencia con lo encontrado en otros estudios vinculados a la temática en relación a su utilidad (Camic, 2008). Por un lado subrayan que la actividad gráfica si presenta efectos sobre variables específicas ya estudiadas en el ámbito de la salud psicológica, el estrés y las emociones, al tenerse cambios estadísticamente significativos en ambas: $\mathrm{Z}=0.00, p=.001, \mathrm{~d}$ $=0.07$ al solicitarle a la gente distraerse de la situación estresante y $Z=2.06$, $p=.007, \mathrm{~d}=0.71$, en el caso de las emociones negativas a un nivel general, dato que se adhiere a la literatura que ubica el manejo de dichas emociones como un mediador entre el estrés y el desarrollo de síntomas psicopatológicos (Berking, Poppe, Luhmann, Wupperman, Jaggi y Seifritz, 2012). Así mismo, permite ubicar qué estrategias de afrontamiento pudiesen estar vinculadas con dichos cambios (pensamiento desiderativo como estrategia frente al estrés, en todos los grupos, $\mathrm{M}=12.05$, D.E. $=5.3, p=.017, \mathrm{n}=20$ ), información que no se ha presentado en estudios anteriores (Read, 2009).

Éste estudio cuenta con la ventaja de situarse dentro de dos marcos teóricos robustos, la teoría transaccional del estrésy la regulación emocional (Szczygiel, Buczny y Bazinka, 2012), lo que permite tanto utilizar instrumentos de medición que cuentan con adecuadas propiedades psicométricas, como la posibilidad de contrastarle con estudios similares y posteriores. Gracias a su diseño, permitió conocer la interacción entre las variables estudiadas (estrés y emociones) con la elaboración gráfica y el abordaje cognitivo de la persona ante aspectos específicos de una situación personal, permitiendo con ello un acercamiento a lo que ocurre usualmente en terapia. Así mismo, al poder ubicar los efectos en la regulación emocional por cada estado afectivo por separado permite su valoración y abre una serie de dudas, primeramente, en torno a las emociones positivas: ¿Qué 
sucede con ellas? ¿Por qué no se vieron modificadas? Como posibilidad, el conocimiento sobre la interacción entre lo saludable y las modificaciones a las emociones negativas, propuesta por Berking, Poppe, Luhmann, Wupperman, Jaggi y Seifritz (2012) salta a la vista. Sin embargo, y una vez adentrándose a las limitaciones del estudio, al tratarse de una única intervención, no se tienen datos que apoyen dicha hipótesis, lo que también deja en duda los cambios en el significado de las vivencias referido en diversos estudios y el peso que tiene la interacción terapéutica en el mismo.Objetivos que se pueden cubrir en estudios con múltiples sesionesperoque requerirían de una evaluación extra en torno a la figura del profesional en salud.

\section{Referencias bibliográficas}

BERKING, M., POPPE, C., LUHMANN, M., WUPPERMAN, P. \& JAGGI, V. (2012). Is association between various emotion-regulation skills and mental health mediated by the ability of modify emotions? Results from two crosssectional studies. Journal of Behavior Therapy and Experimental Psychiatry, 43, 931-937. doi:10.1016/jbtep.2011.09.009

CAMIC, P. (2008). "Playing in the mud: Health psychology, the arts and creative approaches to health care". En Journal of Health Psychology, 13, 2, 287-298.

CANO, F., RODRÍGUEZ, L. Y GARCÍA, J. (2007). "Adaptación española del inventario de estrategias de afrontamiento". En Acta Española de Psiquiatría, 35,1, 29-39. Recuperado de: http://personal.us.es/fjcano/drupal/?q=node/8

CENTER FOR THE STUDY OF EMOTION AND ATTENTION (s.f.). "International Affective Picture System". En http://csea.phhp.ufl.edu/index.html

GONZÁLEZ, M. T. Y LANDERO, R. (2007). "Factor Structure of the Perceived Stress Scale (PSS) in a Sample from Mexico". EnThe Spanish Journal of Psychology, 10,1, 199-206.

READ, D. (2009). "Commentary: Examining underlying paradigms in the creative arts therapies of trauma". EnThe Arts in Psychotherapy, 36, 114-120. doi:10.1016/j.aip.2009.01.011

ROBLES, R. Y PÁEZ, F. (2003). "Estudio sobre la traducción al español y las propiedades psicométricas de las escalas de afecto positivo y negativo (PANAS)". En Salud Mental, 26,1, 69-75. Recuperado de: http://www.medigraphic.com/pdfs/salmen/sam-2003/sam031h.pdf

SLAYTON, S., D'ARCHER, J. Y KAPLAN, F. (2010). "Outcomes studies on the efficacy of art therapy: A review of findings". En Art Therapy: Journal of the American Art Therapy Association, 27, 3, 108-118. doi:10.1080/07421656.2010.10129660

SZCZYGIEL, D., BUCZNY, J. Y BAZINKA, R. (2012). "Emotion regulation and emotional information processing: The moderating effect of emotional 
awareness". EnPersonality and Individual Differences, 52,3, 433-437. doi:10.1016/j.paid.2011.11.005

WOOD, M., MOLASSIOTIS, A. \& PAYNE, S. (2011). What research evidence is there for the use of art therapy in the management of symptoms in adults with cancer? A systematic review. Psycho-Oncology, 20, 135-145. doi:10.1002/pon. 1722 